\title{
Generation of spin-polarized currents in Zeeman-split Tomonaga-Luttinger models
}

\author{
Takashi Kimura, Kazuhiko Kuroki, and Hideo Aoki \\ Department of Physics, University of Tokyo, Hongo, Tokyo 113, Japan
}

(June 11, 2018)

\begin{abstract}
In a magnetic field an interacting electron gas in one dimension may be described as a Tomonaga-Luttinger model comprising two components with different Fermi velocities due to the Zeeman splitting. This destroys the spin-charge separation, and even the quantities such as the density-density correlation involve spin and charge critical exponents $(K)$. Specifically, the ratio of the up-spin and down-spin conductivities in a dirty system diverges at low temperatures like an inverse power of the temperature, $T^{-\left(K_{\uparrow}-K_{\downarrow}\right)}$, resulting in a spin-polarized current. In finite, clean systems the conductance becomes different for up- and down-spins as another manifestation of the electron-electron interaction.
\end{abstract}

72.15-v, 73.20.Dx, 72.10.Bg

Typeset using REVTEX 
Recent studies of quantum transport in mesoscopic systems have brought to light many unusual features unexpected for classical systems [1]. This is heightened by recent advances in fabricating nanostructure quantum wires and quasi-one-dimensional (1D) crystal structures.

In $1 \mathrm{D}$ systems, the interactions between the electrons is so crucial due to the strong constraint in the phase space that the system becomes universally what is called the TomonagaLuttinger (TL) liquid as far as low-lying excitations are concerned no matter how small the interaction may be [2]. A most striking feature of this 1D model is the spin-charge separation. The transport properties [3] are also dominated by the spin-charge separation in the following sense. The low-temperature conductivity of the dirty TL liquid as studied by Luther and Peschel [4] exhibits a power law, $\sigma(T) \sim T^{2-K_{\rho}-K_{\sigma}}$. The power law comes from the degraded Fermi singularity in the TL model, while the critical exponents (which are functions of the interaction) enter as a sum of $K_{\rho}$ for the charge phase of the system and $K_{\sigma}$ (which is actually fixed at 1 for spin-independent interactions) for the spin phase. A recent experiment [7] for high-quality quantum wires seems to support this result. For clean systems Kane and Fisher [5] and Furusaki and Nagaosa [6] found that the conductance quantization in finite systems in the noninteracting case becomes proportional to the exponent, $G=\left(e^{2} / \pi\right) K_{\rho}$ (where $\hbar=k_{B}=1$ is assumed hereafter).

Now we can raise an intriguing question: what happens if we degrade the spin-rotational $(\mathrm{SU}(2))$ symmetry? Such a situation is realized by applying a magnetic field, which makes the Fermi velocities spin dependent due to the Zeeman splitting. In this paper, we show that the spin-charge separation will be then destroyed, causing even the quantities such as the density-density correlation involve spins. Thus the spin may manifest itself in the transport, leading possibly to spin-polarized currents, which is shown to be the case.

The generation of spin-polarized currents has been of a long-standing interest for academic [8] as well as practical points of view, where typical applications include spin-polarized STM [9] and the Mott-detector [10]. Fasol and Sakaki [11] have suggested that in the spinorbit split bands of GaAs quantum wires the curvature in the band dispersion (as opposed 
to the linearized dispersion in the Tomonaga-Luttinger model) will make the relaxation time due to the electron-electron interaction spin-dependent and consequently make the outgoing current spin-polarized. The mechanism proposed in this Letter is by contrast a purely electron-correlation effect, where the ratio, $\sigma_{\uparrow} / \sigma_{\downarrow}$, diverges toward $T=0$.

We start from a clean, two-band Luttinger model, which is similar to the one employed in a study for the Fermi-edge singularity in 1D [12]. The Hamiltonian is given by

$$
H_{\text {clean }}=H_{0}+H_{\text {int }},
$$

where the non-interacting Hamiltonian $H_{0}$ is written as

$$
\begin{aligned}
H_{0}= & \sum_{k, s, i} v_{F s}\left[(-)^{i+1} k-k_{F s}\right] c_{i k s}^{\dagger} c_{i k s} \\
= & \frac{2 \pi}{L} \sum_{q>0} \sum_{i}\left\{v_{0}\left[\rho_{i}(q) \rho_{i}(-q)+\sigma_{i}(q) \sigma_{i}(-q)\right]\right. \\
& \left.+\Delta v\left[\rho_{i}(q) \sigma_{i}(-q)+\rho_{i}(-q) \sigma_{i}(q)\right]\right\} .
\end{aligned}
$$

Here $v_{F \uparrow}\left(v_{F \downarrow}\right)$ is the Fermi velocity of the up (down) spin subband in a magnetic field $B$ with an average $v_{0} \equiv\left(v_{F \uparrow}+v_{F \downarrow}\right) / 2$ and the difference $\Delta v \equiv\left(v_{F \uparrow}-v_{F \downarrow}\right) / 2$. Similarly, $k_{F \uparrow}$ $\left(k_{F \downarrow}\right)$ is the Fermi momentum of the up (down) spin, which equal to $k_{F}$ in the absence of $B$. $c_{i k s}^{\dagger}$ creates a right-going $(i=1)$ or left-going $(i=2)$ electron with momentum $k$ and spin $s$, while $\rho_{i}(q)\left[\sigma_{i}(q)\right]$ are the usual charge [spin] density operators, and $L$ is the system size.

The second line in the above equation indeed indicates that the charge and spin are no longer decoupled for $\Delta v \neq 0$, in a sharp contrast to the usual Tomonaga-Luttinger model.

The spin-charge separated part can be cast into the usual phase Hamiltonian where $v_{0}$ corresponds to the Fermi velocity $v_{F}$. Thus we can introduce the usual phase field $\theta_{+}(x)$ $\left(\phi_{+}(x)\right)$ and the dual field $\theta_{-}(x)\left(\phi_{-}(x)\right)$ corresponds to the charge (spin) degree of freedom to have

$$
\begin{aligned}
H_{\text {clean }}= & \frac{v_{\rho}}{4 \pi} \int d x\left\{\frac{1}{K_{\rho}}\left[\partial_{x} \theta_{+}(x)\right]^{2}+K_{\rho}\left[\partial_{x} \theta_{-}(x)\right]^{2}\right\} \\
& +\frac{v_{\sigma}}{4 \pi} \int d x\left\{\frac{1}{K_{\sigma}}\left[\partial_{x} \phi_{+}(x)\right]^{2}+K_{\sigma}\left[\partial_{x} \phi_{-}(x)\right]^{2}\right\} \\
& +\frac{\Delta v}{2 \pi} \int d x\left\{\left[\partial_{x} \theta_{+}(x)\right]\left[\partial_{x} \phi_{+}(x)\right]+\left[\partial_{x} \theta_{-}(x)\right]\left[\partial_{x} \phi_{-}(x)\right]\right\},
\end{aligned}
$$


where $K_{\rho}\left(K_{\sigma}\right)$ is the critical exponent of the charge (spin) phase. In the following we assume that the coupling constants between electrons do not have insignificant magnetic field dependences, so that we have $K_{\sigma}=1, v_{\sigma}=v_{0}, K_{\rho}=1 / \sqrt{1+4 g}$, and $v_{\rho}=v_{0} / K_{\rho}=v_{0} \sqrt{1+4 g}$, where $g\left(\sim U / 2 \pi v_{0}\right.$ for the Hubbard model $)$ is the dimensionless, forward-scattering coupling constant. Here we have neglected the backward scattering and Umklapp scattering processes, since they have large momentum transfers.

We can diagonalize $H_{\text {clean }}$, as is done for the electron-hole system in a two-channel Tomonaga-Luttinger study of the excitonic phase by Nagaosa and Ogawa [13], via a linear transformation to two new phases,

$$
\left(\begin{array}{c}
\theta_{+}(x) \\
\phi_{+}(x)
\end{array}\right)=\left(\begin{array}{cc}
\cos \alpha & -\frac{1}{y} \sin \alpha \\
y \sin \alpha & \cos \alpha
\end{array}\right)\left(\begin{array}{c}
\tilde{\theta}_{+}(x) \\
\tilde{\phi}_{+}(x)
\end{array}\right)
$$

where $\alpha$ is the 'rotation angle in the spin-charge space' ( $\propto \Delta v$ for small $\Delta v)$ with $\tan 2 \alpha=$ $2\left(\Delta v / v_{0}\right) \sqrt{2\left(K_{\rho}^{-2}+1\right)} /\left(K_{\rho}^{-2}-1\right)$ and $y^{2}=\frac{1}{2}\left(K_{\rho}^{-2}+1\right)$. These new phases also have gapless, linear dispersions, in which the new velocities are given by

$$
\tilde{v}_{\rho, \sigma}^{2}=\Delta v^{2}+\frac{1}{2} v_{0}^{2}\left[K_{\rho}^{-2}+1 \pm\left(K_{\rho}^{-2}-1\right) \sqrt{1+\tan ^{2} 2 \alpha}\right],
$$

where $+(-)$ sign corresponds to $\tilde{v}_{\rho}\left(\tilde{v}_{\sigma}\right)$.

Now we can turn to the calculation of the conductivity in a dirty system. The total Hamiltonian is then $H=H_{\text {clean }}+H_{\text {imp }}$, where the impurity scattering part $H_{\text {imp }}$ is given by

$$
H_{\mathrm{imp}}=\sum_{s} \sum_{l} \int d x N_{s}(x) u\left(x-x_{l}\right),
$$

where $u\left(x-x_{l}\right)$ is the impurity potential situated at $x_{l}$ and $N_{s}(x)$ is the density operator of spin $s$ electrons, whose phase representation is $N_{s}=\frac{1}{2 \pi} \partial_{x}\left(\theta_{+}+s \phi\right)+\frac{1}{\pi \Lambda} \cos \left[2 k_{F s} x+\theta_{+}+s \phi\right]$ with $\Lambda$ being a short-range cutoff. The conductivity of $s$-spin subband, $\sigma_{s}$, is given by $\sigma_{s}=n_{e} e^{2} \tau_{s} / 2 m_{s}^{*}$, where $\tau_{s}$ is the relaxation time of the spin $s$ subband, $n_{i}\left(n_{e}\right)$ is the density of impurities (electrons), and $m_{s}^{*} \propto v_{s}^{-1}$ is the effective mass of the spin $s$ subband. 
In $1 \mathrm{D}$ we have $n_{e}=2 k_{F} / \pi$, but we have neglected the trivial magnetic-field dependence of $k_{F \uparrow}$ and $k_{F \downarrow}$ to single out the effect of differentiated $v_{F \uparrow, \downarrow}$.

We can then calculate $\tau_{s}$ following Götze and Wölfle in the Mori formalism for the conductivity [14] [15], in the second order in $H_{\text {imp }}$ as

$$
\frac{1}{\tau_{s}} \approx 4 \pi v_{F s} n_{i} u^{2}\left(2 k_{F}\right) \sum_{q} \lim _{\omega \rightarrow 0} \frac{\operatorname{Im} \Pi_{s}\left(2 k_{F s}+q, \omega\right)}{\omega},
$$

where $u(q)$ is the Fourier transformation of $u(x)$, and $\Pi_{s}$ is the density-density correlation function.

In terms of the density operator $\rho_{s}(x)$ for spin $s$ we have

$$
\lim _{\omega \rightarrow 0} \sum_{q} \frac{\operatorname{Im} \Pi_{s}\left(2 k_{F s}+q\right)}{\omega}=\frac{1}{2 T} \sum_{s^{\prime}} \int_{-\infty}^{\infty} d t\left\langle\rho_{s}(0, t) \rho_{s^{\prime}}(0,0)\right\rangle .
$$

In the summation over the spin $s^{\prime}$ we can readily show that the cross term, $\left\langle\rho_{\uparrow}(0, t) \rho_{\downarrow}(0,0)\right\rangle$, vanishes. Then the conductivity becomes a sum of the two spin components, each of which has a simple power-law temperature dependence as in the usual Luttinger theory,

$$
\sigma_{s}(T)=\sigma_{0}\left(\frac{v_{F s}}{v_{0}}\right)^{2}\left(\frac{T}{\omega_{F}}\right)^{2-K_{s}},
$$

where $\sigma_{0} \equiv \sigma\left(T=\omega_{F}\right)$ (whose dependence on $k_{F s}$ is again ignored here) and $\omega_{F} \sim \epsilon_{F}$ is the high-energy cutoff.

Here the spin-dependent exponent $K_{s}$ is given by

$$
K_{s}=(\cos \alpha+s y \sin \alpha)^{2} \tilde{K}_{\rho}+\left(\cos \alpha-\frac{s}{y} \sin \alpha\right)^{2} \tilde{K}_{\sigma},
$$

where $\tilde{K}_{\rho(\sigma)}$ are the critical exponent of the phase $\tilde{\theta}(\tilde{\phi})$ given by

$$
\begin{aligned}
\tilde{K}_{\rho(\sigma)}^{2}= & y^{\mp 2}\left[K_{\rho}^{-2}+3 \pm\left(K_{\rho}^{-2}-1\right) \sqrt{1+\tan ^{2} 2 \alpha}\right] \\
& \times\left[3 K_{\rho}^{-2}+1 \pm\left(K_{\rho}^{-2}-1\right) \sqrt{1+\tan ^{2} 2 \alpha}\right]^{-1},
\end{aligned}
$$

where the upper (lower) sign corresponds to $\tilde{K}_{\rho}\left(\tilde{K}_{\sigma}\right)$.

The above equations $(10) \sim(12)$ are the key result of this paper: the electron-electron interaction does indeed make the conductivity dependent on the spin, where the power-law 
dependence in $T$ is retained so that the spin dependence becomes more enhanced at lower temperatures. The ratio $\sigma_{\uparrow} / \sigma_{\downarrow} \propto T^{-\left(K_{\uparrow}-K_{\downarrow}\right)}$ actually diverges toward $T \rightarrow 0$, resulting in a spin-polarized current. This divergence is purely an effect of the electron correlation.

The temperature dependence of $\sigma_{\uparrow} / \sigma_{\downarrow}$ numerically calculated in the presence of a fixed electron-electron interaction $g$ and the several ratios of $v_{F \uparrow}$ to $v_{F \downarrow}$ is displayed in Fig.1. Figure 2 shows a drastic dependence of $\sigma_{\uparrow}\left(\sigma_{\downarrow}\right)$ on the ratio $v_{F \uparrow} / v_{F \downarrow}$ at a fixed temperature with a fixed $g$.

The result shows that the more conductive channel is the spin having a smaller $v_{F}$, since $K_{\uparrow}>K_{\downarrow}$ for $\Delta v \propto\left(v_{F \uparrow}-v_{F \downarrow}\right)>0$. Physically, the effective electron-electron interaction is smaller (larger) in the lighter (heavier) spin subband, since it is the ratio of the electronelectron coupling constant to the kinetic energy $\left(\propto v_{F}\right)$ that matters. Thus the result is roughly consistent with the observation in a single TL liquid that the electron-electron repulsive interaction suppresses the conductivity [4].

If we look more closely for an intuitive interpretation of the present result, one could regard the single TL model of spin 1/2 electrons as a double-chain system of spinless electrons. The present situation could then be regarded as a generalization of our previous model [15], where we have considered two equivalent chains having intrachain and interchain interactions in the absence of interchain tunneling. When the two 'chains' are made inequivalent by the differentiated $v_{F}$, this modifies both the 'intra-chain' (parallel-spin) dimensionless coupling constant, $g / v_{F}$, and 'inter-chain' (antiparallel-spin) $g / v_{F}$. Note that, if the $g$-parameters derive from an $\mathrm{SU}(2)$ symmetric interaction (such as the Hubbard $U$ ) the $g$ 's for intra- and inter-chain interactions are the same. It is then a highly nontrivial question what conductivities will come out. The present result indicates that the chain that has a smaller $g / v_{F}$ does indeed remain more conductive, so that the effect of parallel-spin interaction eventually prevails. This is consistent with the double-chain result that the intra-chain repulsion suppresses the conductivity, while the effect of inter-chain interactions, which incidentally enhances the conductivity, is only of the second order.

Next we consider the conductance of finite, clean systems. We can calculate the conduc- 
tance, $G_{s}$, of spin $s$ subband from the current-current correlation function as [5]

$$
G_{s}=\lim _{\omega \rightarrow 0} \sum_{s^{\prime}} \frac{1}{\omega L} \int d \tau \int d x e^{i \omega \tau}\left\langle T_{\tau} J_{s}(\tau) J_{s^{\prime}}(0)\right\rangle
$$

where $J_{s}=\partial_{\tau}(\theta+s \phi) / 2$ is the spin $s$ current, and $\tau$ is the imaginary time. Then we end up with

$$
G_{s}=\frac{e^{2}}{2 \pi}\left[\left(\cos ^{2} \alpha+\frac{y s}{2} \sin 2 \alpha\right) \tilde{K}_{\rho}+\left(\frac{1}{y^{2}} \sin ^{2} \alpha-\frac{s}{2 y} \sin 2 \alpha\right) \tilde{K}_{\sigma}\right]
$$

Thus the conductance too depends on the spin in contrast to the ordinary case with $G_{\uparrow}=$ $G_{\downarrow}=\left(e^{2} / 2 \pi\right) K_{\rho}$. We may emphasize that the Fermi velocity of the system does not appear in the Landauer formula [16], so that the full expression of the spin-dependent conductance is generated by the electron-electron interaction (while in the conductivity the power of the temperature is). Figure 3 numerically depicts the way in which $G_{\uparrow}\left(G_{\downarrow}\right)$ increase (decrease) with the ratio $v_{F \uparrow} / v_{F \downarrow}$ with a fixed $g$.

Finally let us make a comment on the Anderson localization. In Ref. [17 Giamarchi and Schulz have shown in the absence of magnetic fields that the temperature at which the Anderson localization occurs in $1 \mathrm{D}$ is lower for larger $K_{\rho}$. Combining this result with our present result in the presence of magnetic field, we can envisage a dramatic situation in which the electrons with one spin are Anderson-localized while the others are conductive in a certain temperature region.

We believe these novel many-body effects can be experimentally measured in quantum wires by taking appropriate fillings of the up- and down-spin subbands in a given magnetic field. Unfortunately, in the case of the usual electron-doped GaAs quantum wires the gfactor $(\sim-0.4$ in the bulk) is too small to attain the sufficient Zeeman splitting. However, if we can prepare e.g. InSb quantum wires (whose g-factor is as large as $\sim-50$ in the bulk [18]), the strength of the Zeeman splitting in a typical magnetic field of $1 \mathrm{~T}$ amounts to $\mathrm{g} \mu_{B} H \sim 3.0 \mathrm{meV}$. In such cases a significant deviation of $v_{\uparrow} / v_{\downarrow}$ from unity may be expected.

We are much indebted to Professor Gerhard Fasol for illuminating discussions, and to Professor Tetsuo Ogawa for sending us preprints prior to publication. 


\section{REFERENCES}

[1] See, e.g., Mesoscopic Phenomena in Solid, ed. B.L. Altshuler et al. (North Holland, 1991); Transport Phenomena in Mesoscopic Systems, ed. H. Fukuyama and T. Ando (Springer Verlag, 1991).

[2] See, e.g., J. Sólyom, Adv. Phys. 28, 201 (1979);V.J. Emery in Highly Conducting OneDimensional Solids, ed. by J.T. Devreese et al. (Plenum, New York, 1979), p.247; H. Fukuyamaand H. Takayama in Electronic Properties of Inorganic Quasi-One Dimensional Compounds, ed. by P. Monçeau (D. Reidel, 1985), p.41.

[3] M. Ogata and P.W. Anderson, Phys. Rev. Lett. 70, 3087 (1993); W. Apel and T.M. Rice, Phys. Rev. B 26, 7063 (1982); H. Fukuyama et al., J. Phys. Soc. Jpn 62, 1109 (1993); A. Kawabata, J. Phys. Soc. Jpn.63, 2047 (1994); K.A. Matveev et al., Phys. Rev. Lett. 71, 3351 (1993); M. Fabrizio et al., Phys. Rev. Lett. 72, 2235 (1994); N. Nagaosa and A. Furusaki, J. Phys. Soc. Jpn. 63413 (1994).

[4] A. Luther and I. Peschel, Phys. Rev. Lett. 32, 992 (1974).

[5] C.L. Kane and M.P.A. Fisher, Phys. Rev. Lett. 68, 1220 (1992).

[6] A. Furusaki and N. Nagaosa, Phys. Rev. B 47, 4631 (1993).

[7] T. Tarucha, T. Honda and T. Saku, Solid. State. Commun. 94233 (1995).

[8] V.M. Edelstein, Solid. State. Commun. 73233 (1990).

[9] R. Wiesendanger et al., Phys. Rev. Lett. 65247 (1990).

[10] K. Koike et al., Jpn. J. Appl. Phys. 221332 (1983).

[11] G. Fasol and H. Sakaki, Phys. Rev. Lett. 70, 3643 (1993); G. Fasol and H. Sakaki, Jpn. J. Appl. Phys. 323879 (1994).

[12] T. Ogawa and H. Otani, J. Phys. Soc. Jpn. 64 (1995); T. Ogawa and H. Otani, preprint; H. Otani and T. Ogawa, preprint. 
[13] N. Nagaosa and T. Ogawa, Solid State Commun. 88, 295 (1993).

[14] W. Götze and P. Wölfle, Phys. Rev B 6, 1226 (1974).

[15] T. Kimura et al., Phys. Rev. B 49, 16852 (1994).

[16] R. Landauer, IBM J. Res.\& Dev. 1, 223 (1957).

[17] T. Giamarchi and H.J. Schulz, Phys. Rev. B 37, 325 (1988).

[18] Numerical Data and Functional Relations in Science and Technology-Crystal and Solid State Physics, ed. by O. Madelung et al., Landolt-Börnstein, New Series, Group III, Vol. 16, Pt. a (Springer, Berlin, 1982). 


\section{FIGURES}

FIG. 1. The result for the temperature dependence of the ratio $\sigma_{\uparrow} / \sigma_{\downarrow}$ with a fixed interaction $g=0.5$, and with several ratios of $v_{F \uparrow}$ to $v_{F \downarrow}$.

FIG. 2. The result for the dependence on $v_{F \uparrow} / v_{F \downarrow}$ (keeping $v_{F \uparrow}+v_{F \downarrow}=$ constant) of the conductivity $\sigma_{\uparrow} / \sigma_{0}$ and $\sigma_{\downarrow} / \sigma_{0}$ with a fixed temperature $T=10^{-3} \omega_{F} \sim 100 \mathrm{mK}$ and with a fixed interaction $g=0.5$.

FIG. 3. The result for the dependence on $v_{F \uparrow} / v_{F \downarrow}$ (keeping $v_{F \downarrow}+v_{F \uparrow}$ constant) of the conductance $G_{\uparrow}$ and $G_{\downarrow}$ normalized by $e^{2} / \pi$ with a fixed interaction $g=0.5$. 